\title{
A Rate Control Technique for Off Line H.264/AVC Video Coding Using Subjective Quality of Video
}

\author{
S. L. P. Yasakethu, W. A. C. Fernando, Senior Member IEEE, S. Adedoyin and A. Kondoz, Member IEEE
}

\begin{abstract}
In this paper, we propose a rate control technique for H.264/AVC using subjective quality of video for off line video coding. We propose to use Video Quality Metric (VQM) with an evolution strategy algorithm, which is capable of identifying the best possible quantization parameters for each frame to encode the video sequence such that it would maximize the subjective quality of the entire video sequence subjected to the target bit rate. Simulation results suggest that the proposed technique can improve the $R D$ performance of the H.264/AVC codec significantly. With the proposed technique, up to $35 \%$ bit rate reduction can be achieved at the same video quality. Furthermore, results show that the proposed technique can improve the subjective quality of the encoded video significantly for video sequences especially with high motion.
\end{abstract}

Index Terms - Video coding, H.264/AVC, subjective quality

\section{INTRODUCTION}

Unprecedented growth of consumer apatite for multimedia contents has become the major driving force governing today's dynamics of the consumer electronic market. Due to the ever falling cost, multimedia enabled consumer electronic devices are within the reach of the most of the global population and therefore, they are no longer considered as luxury devices. Consequent sheer demand for consumer electronic devices, in return, paves the way to new technological innovations and advancements within the consumer electronic domain that reduces the cost even further while including more demanding functionalities to existing devices. While this tremendous technological innovations and growth of consumer demand touches every corner of the consumer electronic domain, multi-functional portable devices has won unimaginable attention. The best example is fordable consumer video devices such as DVD players. Inline with these market dynamics, the demand for more cost effective multimedia enabling technologies with efficient usage of system resources such as bandwidth and memory space, are going to be very important in the future.

Video coding technologies have evolved significantly during the past decade, dominated by the work on ISO/IEC MPEG and ITU-T H.26x standards based techniques. These approaches were characterized by a highly complex video encoder structure and a significantly lower complex decoder structure as demanded by many popular applications involving one-to-many topologies. Video capturing and

This work was supported in part by the European Commission IST FP6 program, under VISNET II project, a European Network of Excellence.

Authors are with the Centre for Communication System Research,

University of Surrey, Guildford, GU2 7XH, UK (e-mail:

\{S.Yasakethu, W.Fernando, S.Adedoyin, A.Kondoz\}@surrey.ac.uk). encoding was conventionally limited compared to the largely spread listeners. In all these work, all encoders used objective based quality metrics to evaluate the video quality. They are simple to calculate and are mathematically easy to deal for optimization purposes. However, it is well-known that these metrics are not suitable to describe the subjective degradation perceived by a viewer. Even though these metrics provide a simple way to evaluate the video quality, they are not well adapted for assessing the quality of the image as perceived by the human visual system. Another drawback of these metrics is that they evaluate the original image with its counterpart encoded/decoded image. They are not adapted to evaluate the temporal relation existing in a video sequence. Therefore, we believe that video coding based on subjective quality assessment produces better optimized results since they are based on actual human perception.

The objective of this paper is to present a novel ratecontrolling algorithm based on Evolution Strategy (ES) for off-line H.264/AVC video encoding by considering subjective quality of the encoded video. The rest of the paper is organized as follows, in section II, an overview of the related works in literature is presented. In section III, we present the proposed solution. Results from our experiments and potential applications of the proposed technique are presented in sections IV and V respectively. Finally, section VI presents the conclusion and future works.

\section{RELATED WORK}

H.264/AVC video compression scheme utilizes motion compensation, differential pulse code modulation and transform coding to perform compression with the help of quantization and variable length coding (VLC) [1]. The trivial problem of video encoders based on these is that their output bit rate fluctuates over the video sequence. This is due to the variation of statistical properties in the encoded video sequence and the different picture types with different compression ratios. Even though variable bit rate (VBR) applications can accommodate for such data rate deviations, for constant bit rate (CBR) applications such as in video storage, bursty data rates cannot be tolerated. A First-In-FirstOut (FIFO) buffer with a feed back mechanism at the encoder output is used to solve this problem. The buffer accepts the bursty encoded data stream and then produces a smooth data stream as its output. The encoder monitors the state of the buffer continuously through a feedback mechanism, according to which, the encoder adjusts the output data rate by controlling the quantization step size to prevent the buffer over flow or under flow errors. A number of authors have 
proposed improved algorithms to optimize the performance of the encoding phenomenon based on several rate distortion concepts [2][3][4]. These algorithms have concentrated on using objective statistical quality measurements for rate distortion optimizations. But it is a well known fact that simple statistical measurements such as mean average difference (MAD) and mean square error (MSE) do not necessarily accurately model perceptual quality due to their lack of correlations with the human visual system (HVS) characteristics [5]. Ultimately, when it comes to the quality of experience of the end-user, the visual quality of the encoded video sequence becomes the most significant feature. However, manipulating the quantization step size to control the data rate directly affects the perceived picture quality. For example higher quantization step size will lead to a poor visual quality and vice versa. Therefore deciding on the best quantization parameters to maximize the perceived video quality subjected to a targeted bit rate is a challenging problem.

Rate control algorithms proposed by different authors can be categorized into two major categories based on whether they are predictive or pre-analytical. Predictive algorithms determine the quantization settings for each macroblock based on the current macroblock and previously encoded macroblocks. In contrast, in delayed-decision pre-analysis algorithms quantization settings may be based on future macroblocks or future frames. Delayed decision scheme can be further divided into two classes; single or multiple frames delayed.

\section{A. Predictive rate control schemes}

In this scheme, the value of the quantization parameter, mquant [6] is determined based on the buffer occupancy level. This method is known as direct buffer-state feedback scheme. This control strategy is applied in macroblock level. Thus, it is called a local control scheme. This strategy results in a quality variation within the frame. Global control scheme can alternatively be used to eliminate this problem. Such schemes assign a single control parameter for each frame. The value of mquant can be kept constant or change adaptively but independent of the rate control in order to improve the visual quality. One good example of a predictive rate control scheme is MPEG Test Model 5 (TM5)[7], in which bit rate is controlled by the value of mquent assigned to each macroblock. mquent is an integer valued variable which lies between 1 and 31 . This algorithm has no particular mechanism to prevent buffer overflowing and under flowing. Three different buffer types are used each picture type instead of single buffer. Hence the control may fail, especially when the buffer size is small. Another drawback in TM5 scheme is that the algorithm does not guarantee Video Buffer Verifier $(V B V)$ [6] compliance. Therefore resulting bit stream may have VBV overflows as well as underflows. These limitations justify the need of further research to formulate a better rate control algorithm. Number of authors has proposed improved algorithms to optimize the performance of the TM5 algorithm based on rate distortion concepts $[2][4][8]$.

\section{B. Delayed-decision bit rate control}

The quantization parameters are determined after analyzing one or more frames of the video sequence. Hence, a better quality can be achieved. Most of the researches on rate control belong to this category and most of them pre-analyze a single frame or a Group of Pictures (GOP). For the single frame delay case, measuring the frame block activities from the current frame before it is encoded, rather than depending on the value calculated for the previous picture can directly improve the TM5 algorithm. Another TM5 based method uses a constant $q$ to quantize and encode all the macroblocks in the frame to get a bit-usage profile, and then uses the profile during the real encoding phase to get better prediction and control on the rate. Another method measures the entropy and uses it to predict the bits at macroblock level to get better control on the rate.

A common problem of these algorithms is that, the quality, or the distortion, is not measured and controlled explicitly. To cope with this problem, another class of rate control methods has been proposed which take the distortion into account. The goal of these methods is to determine the best achievable quality for a given channel rate and buffer size, if the entire video sequence or part of its, future frames are known in advance.

The objective of the bit rate optimization in the context of video coding is to encode the video sequence with the maximum possible decoded video quality under the given bit budget. This problem can clearly be reduced to a constraint optimization problem. Various techniques have been proposed to solve this problem. In [4][9] Lagrange and minimax techniques are proposed to get the optimum picture quality. However, the major disadvantage of these techniques is the sub-optimality in the bit rate-picture quality trade off. Even through the true global optimal performances can be obtained with the trellis-based algorithm proposed by [8] its use is extremely limited due to the heavy coding complexity of the algorithm since it traverses all the quantization steps.

\section{Rate controlling in H.264/AVC}

One of the latest standards in video coding is called H.264 (also called MPEG-4 AVC, Advanced Video Coding defined in MPEG-4 Part 10)[10]. Compared with previous standards, H.264 achieves up to $50 \%$ improvement in bit rate efficiency. It has been adopted by many application standards such as HD DVD, DVB-H, HD-DTV, etc. Therefore, its implementation is a very popular research topic to date.

The rate control algorithm used in H.264/AVC dynamically adjusts encoder parameters to achieve a target bit rate. It allocates bit budget for each group of pictures, individual picture and subpicture in a video sequence. Rate control is not a part of the H.264 standard, but the standards group has issued nonnormative guidance to aid in implementation. Block based hybrid video coding schemes such as H.264 and MPEG-4 are considered to be lossy processes. They try to achieve compression not only by removing the redundant temporal and spatial information from the video sequence but also attempt to 
make quality compromises in ways that would have minimum effects on perceived quality. In particular the quantization parameter/step size (QP) regulates the amount of spatial detail retained. For example when QP is small, much of the information is saved and when QP is large much of the information is discarded to reduce the bit rate at the price of increase in distortion. Therefore the decision on which the trade-off between the visual quality of the encoded video sequence and its encoded bit rate plays a significant role when comes to video applications.

Open loop, variable bit rate encoding mechanism is illustrated in Figure 1. The uncompressed video and the QP value is supplied by the user. As the source video is encoded a fairly constant quality video sequence is obtained, but the bit rate may vary dramatically. This is due to the complexity of the picture been continually changed in a real video sequence. But in reality constrains such as network bandwidth, decoder buffer size and storage capacity forces the video to be encoded at a more nearly constant bit rate. Close loop rate control mechanism used for such constant bit rate applications is shown in Figure 2. To achieve a more or less a constant bit rate, QP values must be dynamically varied based upon estimates of the source complexity such that each picture gets an appropriate amount of bits to work with. Therefore rather than specifying a QP value at the input, the user specifies the target bit rate of the application.

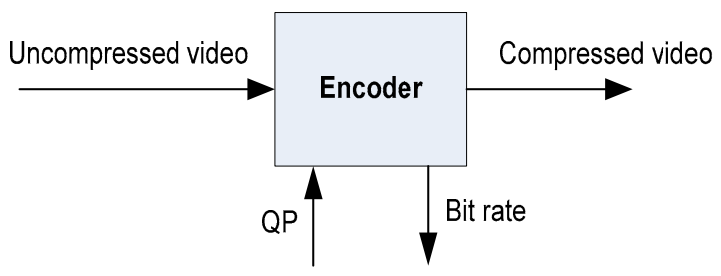

Fig. 1. Open loop encoding mechanism

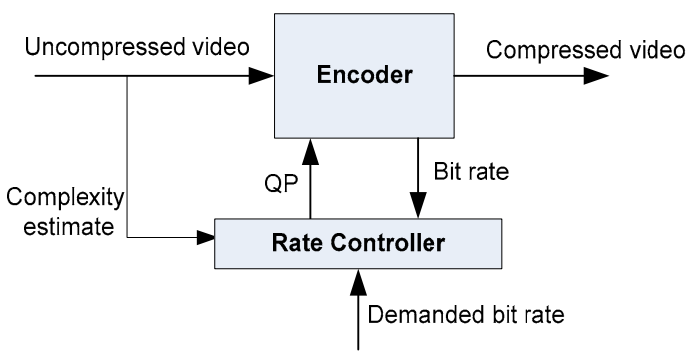

Fig. 2. Close loop rate control mechanism

The heart of the rate-controlling algorithm is the evaluation of the relationship between QP, bit rate and the encoding complexity. Since the QP can only influence the information carried in the residual, the bits and the complexity terms are only associated with the residuals. To evaluate the above relation mean average difference (MAD) of the prediction error is used for the encoding complexity. The mean average difference is defined as shown in equation (1), where $i$ and $j$ refers to the pixel locations of the image.

$$
M A D=\sum_{i j} \mid \text { residual }_{i j}\left|=\sum_{i j}\right| \text { source }_{i j}-\text { prediction }_{i j} \mid
$$

The rate control algorithm uses the MAD value and evaluates the residual bits as shown by equation (2).

$$
\text { ResidualBits }=\left[\frac{C_{1} \times M A D}{Q P}\right]+\left[\frac{C_{2} \times M A D}{Q P^{2}}\right]
$$

The coefficients $C_{1}$ and $C_{2}$ are estimated empirically, by providing hooks in the encoder for extracting the residual coefficients. Having established the model shown in equation (2), the $Q P$ value needed for encoding could be determined, when the target amount Residual Bits is determined.

\section{Evolutionary Strategies}

Evolutionary computation (EC) theories were developed originally from observing natural evolution of life form. Because of this, the terminology surrounding the field of EC is full of analogies with natural evolutionary process. It was particularly from Darwin's theories [19] that the best techniques regarding the optimization, modeling and the control of unknown processes were developed. EC has long been exploited in the video-coding field. A very well known form of EC called Genetic Algorithm (GA) was used to perform image registration as part of a larger Digital Subtraction Angiography (DAS) system [20]. Subsequently, GA search algorithm has been applied for motion estimation [21][22]. Hardware implementation of Four-Step genetic search algorithm was proposed in [21].

Similarly Evolutionary Strategies (ES) were developed to solve complex optimizations problem by Rechenberg and Schwefel in the 1960's [17]. It employs simple algorithmatic methods based on adding random noise to each solution. Due to Schewel research a commonly based method was created that used a mutation mechanism that employs adding a number from a Gaussian distributed curve with expectation of zero mean and a given variance. Rechenberg further developed the algorithm introducing the $(1+\lambda)[18]$, which later transitioned to the $(\mu+\lambda)$. This notation characterizes the selection mechanism. The best individuals out of the union of parents and offspring survive.

\section{Proposed SOlution}

Most pictures, still or moving, are meant to be viewed by people ultimately. Accordingly, image processing, transmission, or synthesis systems should be tailored to the properties of human visual perception. When comes to quality of experience (QoE) of video applications, viewers do not bother about how the application is prepared, they are more concerned about the level of visual quality that they experience. Therefore the decision on which the trade-off between the visual quality of the encoded video sequence and its encoded bit rate plays a significant role when comes to CBR video applications. But currently used rate controlling techniques do not take in to account the HVS parameters when they evaluate suitable QP values for encoding. Since they use statistical measurements similar to the one presented in equation (1) where it only examines the difference in pixel values, the properties of the HVS is not considered. 
Furthermore these techniques are based on pre frame/macroblock basis and QPs per each frame/macroblock is assigned accordingly. But ultimately user visual quality evaluation is based on the entire visual quality of the video rather than a frame/macrablock basis. If quality estimation is carried out frame/macroblock basis it could easily mislead the true visual experience of the user. Therefore the human judgment on visual quality would be more accurate if the subjective quality of the entire video sequence could be evaluated at the encoder, for particular levels of quantization per frame/macroblock, subjected to a target bit rate defined by the application. In this paper, we focus on off-line video coding applications such as storages, VCDs, DVDs where the more emphasis is given to maximizing subjective quality, subject to certain application limitations (e.g. storage capacity).

Numbers of subjective quality metrics have been proposed by different authors in the literature such as Video Quality Mertic (VQM)[11], Perceptual Video Quality Measure (PVQM)[12], and Moving Picture Quality Metric (MPQM)[13]. We propose to use Video Quality Metric (VQM) developed by the Institute of Telecommunication Sciences (ITS) to be used in our technique to subjectively assess the video quality and to evaluate its correlations with the encoding parameters. VQM is a standardized method of measuring video quality that closely predicts the subjective quality ratings that would be obtained from a panel of human. Due to its excellent performance in the International Video Quality Expert's Group (VQEG) Phase II validation tests [14], the NTIA/ITS VQM methods were adopted by the ANSI as a U.S. national standard and as international ITU Recommendations in 2004 [15]. VQM measures the perceptual effects of video impairments including blurring, jerky/unnatural motion, global noise, block distortion and colour distortion, and combines them in to a single metric. The technology is covered by four U.S. patents owned by NTIA/ITS [15].

The proposed methodology for modifying the rate control mechanism for off-line video encoding, incorporating HVS parameters to optimize the encoding mechanism is discussed in this section. Figure 3 illustrates the proposed technique. Rate control problem has been defined using the subjective quality of the output video (VQM) and the target and actual encoded bit rates. We propose a novel Evolution Strategy based algorithm to evaluate the optimum solution to maximize the encoded subjective quality subjected to the bit rate constrained defined by the application.

\section{A. Proposed Evolutionary Strategy (ES)}

Search points in ES represent an N-dimensional object parameter of vectors. With each search point representing Quantization Parameters for each frame of the video sequence. ES typically uses deterministic selection in which the worst solutions are purged from the population based directly on their fitness function value. The $(\mu+\lambda)$-Evolutionary Strategy demonstrated in Figure 4 is used in this work with an increasing level of imitation of biological evolution[23], where $\mu$ means the total number of parents in previous population, and $\lambda$ stands for the number of offspring generated from mutated parents.

\section{B. Chromosome Representation}

$\mathrm{N}$-dimensional object vectors represent a chromosome. This is determined by the video sequences length. Each object vector represents a quantization value within the range of [1 - 51]. Each object vector has two parameters associated to it as shown in equation (3). Equation (4) shows a real valued representation of the chromosome and each element associated strategy parameter. The object parameter $\bar{x}$ represents the actual value of the quantization parameter and the strategy parameter $\bar{\sigma}$ represents the mutation step size. Larger values of the strategy parameter force a large change in the quantization size for a given frame while small changes causes more localized search. The strategy parameter takes the value from $1-n$ and is user defined. The importance of a chromosome in a population is defined by the fitness function.
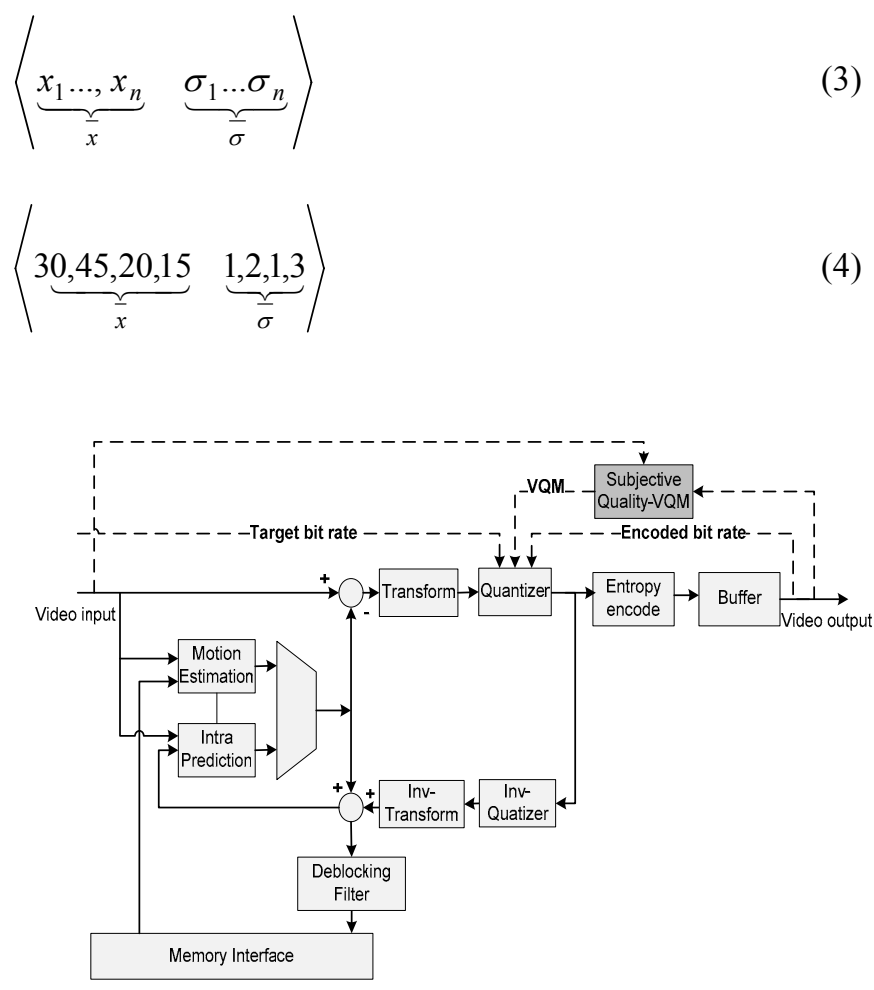

Fig. 3. Proposed technique embedded to the H.264/AVC Codec

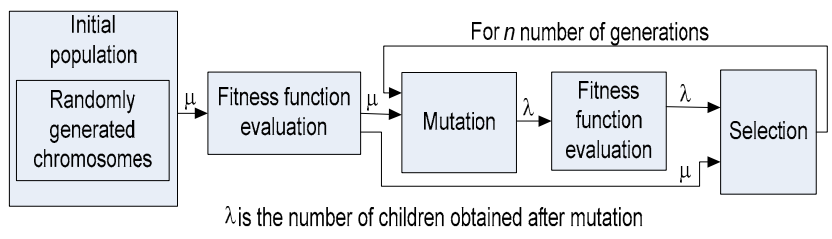

Fig. 4. $(\mu+\lambda)$-Evolutionary Strategy-based algorithm

\section{Population Generation}

Members of the population are made up of randomly generated chromosomes. The initial population size is set to 3 and the number of offspring produced in each generation is fixed to 6 individuals (1:2 mutation ratios). These along with parents are used to select the parents for the next generation Individuals deemed unfit are purged from the population before any new offspring's are produced. 


\section{Fitness Function}

The fitness function divides the strong from the weak and is the driving force of the selection mechanism. The chromosomes with the strongest fitness values are selected to reproduce offspring.

The fitness function is based on,

Fitness $=\left[1-\left(\frac{\text { encoded bitrate }}{\text { traget bitrate }}\right)\right]-\lambda[V Q M]$

In equation (5), target bit rate is specified by the off-line video coding application, where as encoded bit rate and the VQM value relates to the properties of the random individuals according to their encoded parameters. $\lambda$, defines the weightage or else the significance between the encoded bit rate and the VQM score with respect to the application. The value of $\lambda$ is experimentally decided, allowing for fast convergence of the algorithm to its termination criteria discussed below. A VQM score of 0 implies the best visual quality and 1 implies the worst with respect to the original video. For quality evaluation VQM takes the entire encoded sequence where by more, accurate user experience could be estimated at the encoder.

\section{E. Mutation}

The mutation stage is the decisive part in generating new offspring. It is based on Gaussian distribution with mean $\varepsilon$ and standard deviation $\sigma$. The mean is always set to zero and the distribution is symmetric to this, thus allowing the possibility of drawing a random number with a given magnitude to be a rapidly decreasing function of the standard deviation $\sigma$. Thus the value of $\sigma$ value is a parameter of the algorithm that determines the extent to which given values of $x_{i}$ are perturbed by the mutation operator. The mutation step size also undergoes mutation and this mechanism is specified by the following formula. Where $T$ is the learning

$$
\sigma^{\prime}=\sigma \times e^{T \times N(0,1)}
$$

rate and $N(0,1)$ represents a number drawn from a Gaussian distribution with mean 0 and standard deviation 1 . The mutated $\sigma^{\prime}$ is then multiplied by $N_{i}(0,1)$. This value is then added to object parameter to produce a new offspring $x_{i}{ }^{\prime}$ defined by equation (7).

$x_{i}^{\prime}=x_{i}+\sigma^{\prime} \times N_{i}(0,1)$

Where $N_{i}$ is a new draw from the Gaussian distribution. To stop the algorithm producing standard deviations close to zero the following rule is enforced.

$$
\sigma^{\prime}=<\varepsilon_{0} \Rightarrow \sigma^{\prime}=\varepsilon_{0} \text { Where } \varepsilon_{0}=1
$$

Allowing $\sigma$ to be controlled by the algorithm coevolves both the strategy parameter and the solution. This allows each individual frame to have its own mutation operator. Thus, if a frame already has a suitable QP value the strategy parameter will evolve to small number to refine the QP value.

\section{F. Termination}

The algorithm was terminated when an individual meets the flowing criteria.

$$
\text { if }\left[\begin{array}{l}
\text { encoded bitrate } \leq \text { traget bitrate } \\
\& V Q M \leq V Q M_{\text {threshold }}
\end{array}\right]
$$

A numerical value for $V Q M_{\text {threshold }}$ is determined by observing the each video sequence for different encoded levels. If the algorithm fails to find an individual to meet the above criteria the algorithm is terminated after 15 generations to avoid additional delay.

\section{Simulation Results}

Simulations of the proposed ES rate control mechanism is carried out for three test video sequences, Soccer (high motion), Foreman (medium to low motion) and Claire (low motion), to validate the proposed technique for different motion levels. The simulation test conditions are first explained and then the results are analyzed with the state of the art in conventional video coding.

The test sequences are in YUV 4:2:0 sub-sampling format, with QCIF resolution $(176 \times 144)$ and a frame rate of $30 \mathrm{fps}$ within a GOP structure of IPPP. Results are compared with the H.264/AVC codec [16], which is simulated in IPPP GOP structure (JM10.1/Baseline profile). The value of $\lambda$ in the fitness function was experimentally decided and set to 4 for all simulations. Target output bit rate was varied according to the video sequence and it was fixed for both H.264/AVC and for the proposed technique.

\section{A. RD Performance}

Figure 5-10 represent the simulation results for the proposed solution and for the H.264/AVC under similar test conditions. H.264/AVC encoded video sequence has been used to generate its VQM output together with the original video sequence to present its $\mathrm{RD}$ performances. Both subjective (VQM) and objective (PSNR) results are presented to analyze the performance of the algorithm. Results clearly suggest that the proposed rate control technique produces significant improvements compared to the H.264/AVC. Up to $35 \%$ bit rate reduction is possible with the proposed technique for similar subjective quality against the H.264/AVC codec. Results further suggest that the proposed technique can improve the subjective quality of the video output significantly for video sequences with high motion compared to the H.264/AVC. This is due to the selection of the quantization parameters based on the subjective video quality, which will play a main role for such sequences.

In Figures 11 and 12 visual images for 4 consecutive frames for Foreman test sequence are presented at the same visual quality for both the rate controlling algorithms considered. Respective encoded bit rate values shows that the proposed algorithm can significantly reduce the bit rate while keeping the same visual quality. 


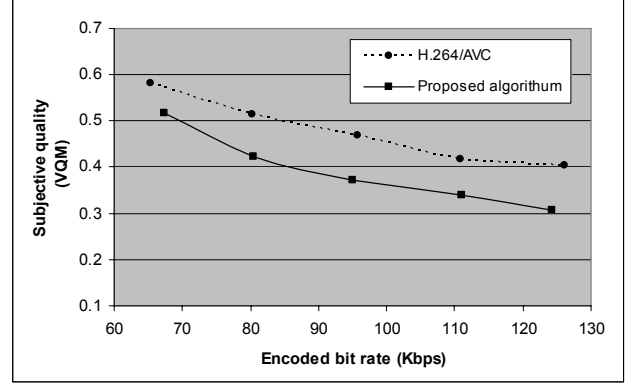

Fig. 5. Subjective quality and encoded bit rate comparison for the Soccer sequence

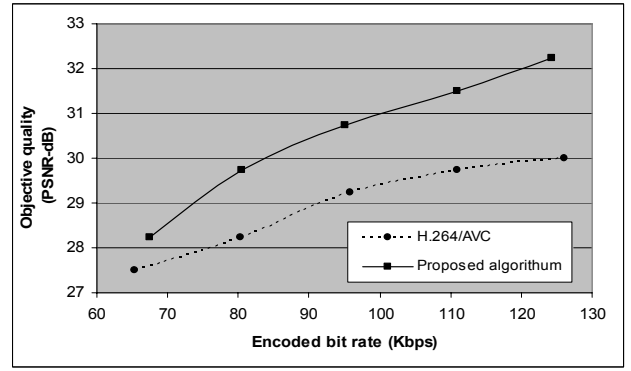

Fig. 6. Objective quality and encoded bit rate comparison for the Soccer sequence

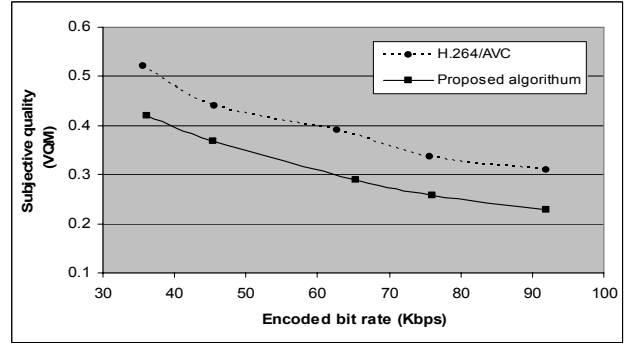

Fig. 7. Subjective quality and encoded bit rate comparison for the Foreman sequence

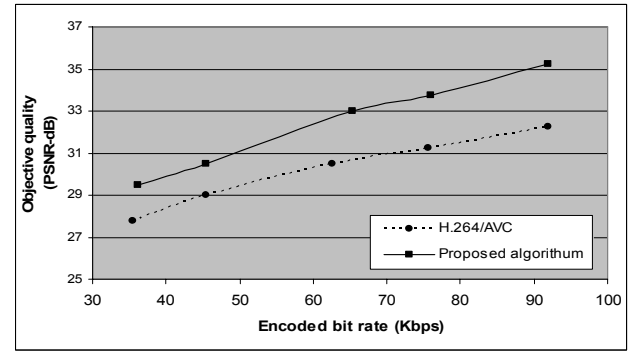

Fig. 8. Objective quality and encoded bit rate comparison for the Foreman sequence

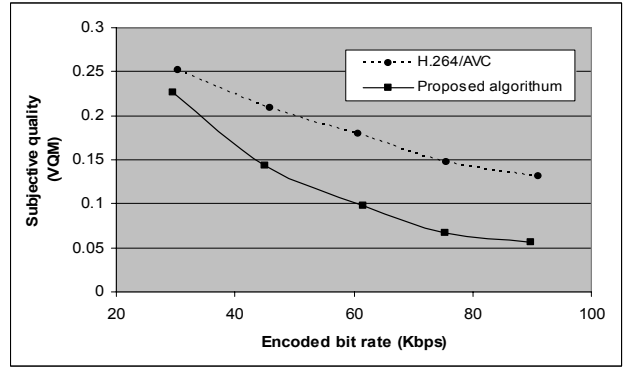

Fig. 9. Subjective quality and encoded bit rate comparison for the Claire sequence

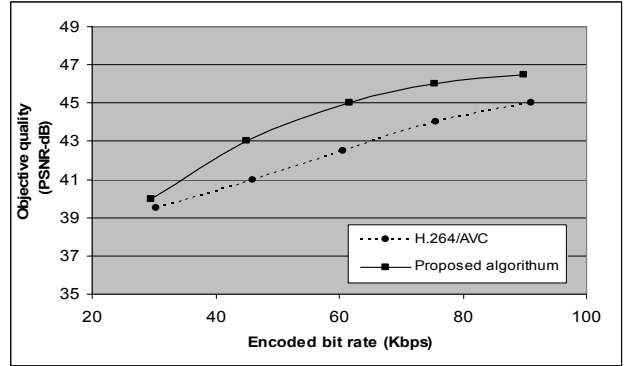

Fig. 10. Objective quality and encoded bit rate comparison for the Claire sequence

\section{B. Target bitrates and encoded bit rates}

Table 1 presents the target and encoded bit rates for the Soccer sequence using both schemes. It clearly shows that the proposed technique provides a bit rate, which is closer to the target bit rate as in H.264/AVC. However, this is achieved at a very high subjective quality. Therefore, the proposed technique can maintain the target bit rate at a higher visual quality than H.264/AVC.

TABLE 1

COMPARISON BETWEEN THE ENCODED BIT RATE AND THE TARGET BIT

\begin{tabular}{|l|l|l|l|}
\multicolumn{4}{|c}{ RATE (FOR THE SOCCER SEQUENCE) } \\
\hline \multirow{4}{*}{ Technique } & $\begin{array}{l}\text { Target bit } \\
\text { rate } \\
(\mathrm{kbps})\end{array}$ & $\begin{array}{l}\text { Encoded } \\
\text { bit rate } \\
(\mathrm{kbps})\end{array}$ & VQM \\
\hline H.264/AVC & 65 & 65.3 & 0.58 \\
\cline { 2 - 4 } & 80 & 80.3 & 0.52 \\
\cline { 2 - 4 } & 95 & 95.8 & 0.47 \\
\cline { 2 - 4 } & 110 & 110.9 & 0.42 \\
\cline { 2 - 4 } & 125 & 125.1 & 0.41 \\
\hline Proposed & 65 & 65.42 & 0.51 \\
\cline { 2 - 4 } & 80 & 79.4 & 0.44 \\
\cline { 2 - 4 } & 95 & 94.7 & 0.37 \\
\cline { 2 - 4 } & 110 & 109.6 & 0.34 \\
\cline { 2 - 4 } & 125 & 124.3 & 0.31 \\
\hline
\end{tabular}

\section{Complexity}

The proposed technique needs some additional computational power compared to the H.264/AVC. This additional power is mainly due to the VQM calculations since VQM has not yet been fully optimized. Other than that the proposed ES algorithm is not computationally expensive since the operations within ES are quite simple. Furthermore, the proposed technique introduces some delay compared to the H.264/AVC. This is again mainly due to the VQM calculations since it needs to buffer few frames. This additional computational power and the delay can be justified since this will be done only once and when it is optimized, we can reduce the bit rate (or memory for storage) significantly, if the proposed technique is used. Thus the proposed technique is suitable for any off-line video coding applications such as storages, VCDs, DVDs and off-line video encoding for streaming, Video on demand applications, broadcasting, where the more emphasis is given to maximizing subjective quality, subject to certain application limitations (e.g. storage capacity, bit rate). 
It should be noted that the proposed technique do not change the complexity of the H.264/AVC decoder. Therefore, the proposed technique is compatible with the existing video decoders such as H.264/AVC based DVD players, digital video receivers, etc.

\section{APPLICATIONS OF THE PROPOSED TECHNIQUE}

In this section, we describe the applications of the proposed technique in the consumer electronics applications.

\section{A. Video On demand (VOD)}

Video on Demand service represents a new opportunity to monetize existing infrastructure, currently used for voice, data or broadcast services. Delivery of television, movies, media, advertising or other popular content to a set-top box through a broadband, wireless or satellite network connection also helps service providers round out their "triple-play" service offering, and to remain competitive in a rapidly evolving marketplace. With the continued global growth of broadband access, Video on Demand services quickly follows. This revolutionary service transforms the viewer's experience by allowing not only "time shifting" - choosing when to watch, but can also eliminate many of the regional, national or global limits of the traditional broadcast cable or satellite architecture. Video on Demand allows service providers to offer an endless array of specialty content to consumers who are willing to pay to watch what they want, when they want it. For these applications storage capacity and the transmission bandwidth play a very important role to maximize the revenue and provide a good quality of service. The proposed algorithm is well suited for these applications since it reduces both the storage capacity and the transmission bandwidth for a given video stream at the same video quality.

\section{B. $D V D / V C D$}

$\mathrm{DVD} / \mathrm{VCD}$ players are very common in the consumer electronics market nowadays. In fact portable DVD players and DVD tablets are very popular among the young generation. The proposed algorithm can also play a very important role in off line video encoding for DVD/VCDs to maximize the storage capacity of the discs.

\section{Other Applications}

The proposed technique can be used for any other off line video coding applications such as off line video programs for broadcasting, advertisements and personal video storage devices.

\section{CONCLUSIONS}

In this paper, we proposed a rate control algorithm based on subjective quality of the video for H.264/AVC in off line video encoding. Simulation results are presented for three different motion video sequences and they clearly suggest that the proposed technique can outperform the RD performance of the state of the art H.264/AVC by a significant margin. Up to $35 \%$ bit rate saving can be achieved with the proposed technique at the same video quality. Unlike rate controlling algorithms, which are based on objective quality of video, the proposed technique can guarantee the subjective quality of the video output at a much lower bit rate. Even though the proposed technique needs some additional computational power, this can be easily justified since the encoding is only done once and the RD performance gain achieved by the proposed technique. In future, we plan to extend this work for on line video encoding.

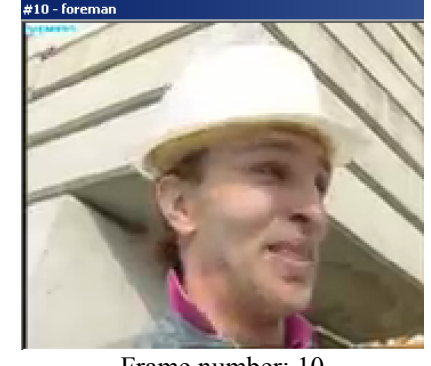

Frame number: 10

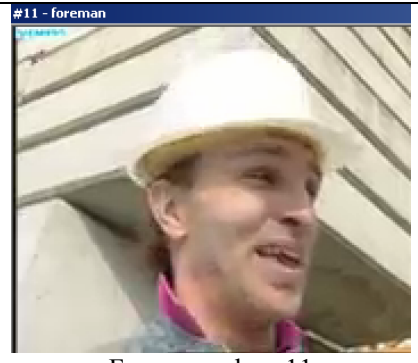

Frame number: 11

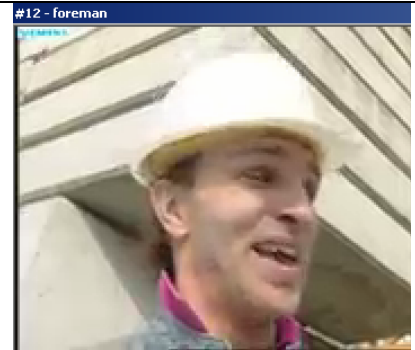

Frame number: 12

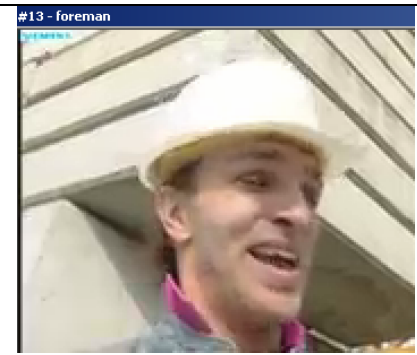

Frame number: 13

Fig. 11 Foreman seq. encoded with proposed algorithm for a bit rate of $60.5 \mathrm{Kbps}, \mathrm{VQM}=0.3$

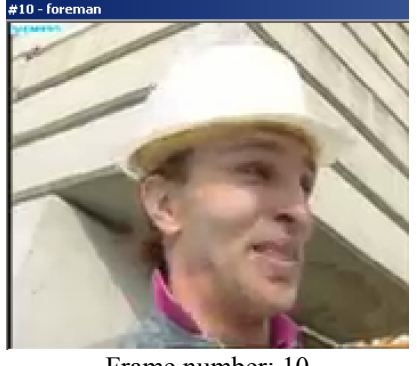

Frame number: 10

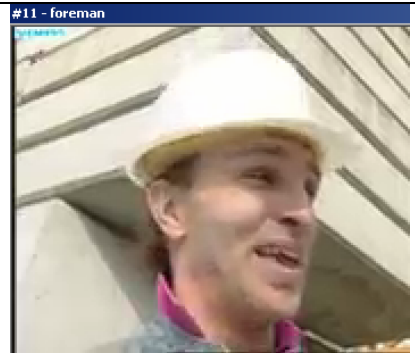

Frame number: 11

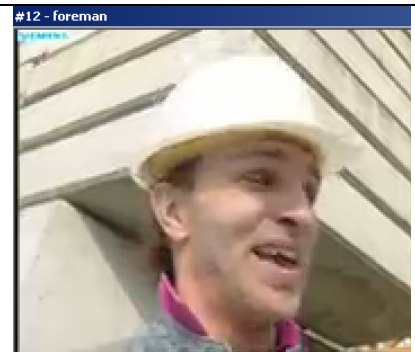

Frame number: 12

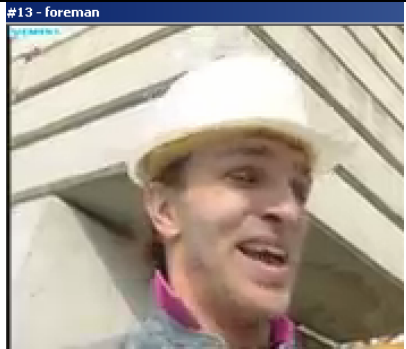

Frame number: 13

Fig. 12 Foreman seq. encoded with H.264/AVC for a bit rate of $91.9 \mathrm{Kbps}, \mathrm{VQM}=0.3$ 


\section{REFERENCES}

[1] Mohammed Ghanbari, Video coding: an Introduction to standard codecs, The Institution of Electrical Engineers, London UK, 1999.

[2] H. Kodikara Arachchi, W.A.C. Fernando, "POS based bit rate optimization for MPEG-1/2 video coding", IEEE International Conference on, Image Processing, 2005. ICIP 2005, Genova 14 Sept. 2005.

[3] H. Kodikara Arachchi, W.A.C. Fernando, "An intelligent rate control algprithm to improve the video quality at scene transitions for off-line MPEG-1/2 encoders", IEEE Transactions on Consumer Electronics, Volume: 49, Issue: 1, pp 210-219, Feb.2003.

[4] L.-J Lin, A. Ortega, and C.-C.J Kuo, "Gradient-based buffer control technique for MPEG", in Proc. of SPIE Visual Communications and Image Processing '95, Taipei, Taiwan, 1995.

[5] Girod, B., "What's wrong with Mean-Squared Error", Digital Images and Human Vision, A. B. Watson Ed., Chapter 15, pp. 207-220, MIT press, 1993.

[6] International Organisation for Standardisation (ISO/IEC JTC1 SC29 WG11), International Standard ISO/IEC 13818, 1996.

[7] ISO/IEC/JTC1/SC29/WGII, “Test Model 5”, Draft, April 1993.

[8] A. Ortaga, K. Ramchandran, and M. Vetterli, "Optimal trellisbased buffered compression and fast approximation", IEEE Trans. Image Proc., Vol. 3, pp. 26-40, Jan. 1994.

[9] L.-J Lin, A. Ortega, and C.-C.J Kuo, "Rate control using splineinterpolated R-D characteristics", in Proc. of SPIE Visual Communications and Image Processing '96, (Orlando, FL), pp. 111122, Mar. 1996.

[10] "Draft ITU-T recommendation and final draft international standard of joint video specification (ITU-T Rec. H>264 | ISO/IEC 14496-10 AVC), "JVT G050, 2003.

[11] M. Pinson and S. Wolf, "A new standardized method for objectively measuring video quality," IEEE Transactions on Broadcasting, v. 50, n. 3, pp. 312-322, Sept. 2004.

[12] A. P. Hekstra et al.: "PVQM- a perceptual video quality measure" Signal Processing: Image Communication 17 (10): 781-798, 2002.

[13] C. J. Branden Lambrecht and O.Verscheure. "Perceptual Quality Measure using a Spatio-Temporal Model of the Human Visual System," Proc. SPIE vol. 2668, p.450-461, March, 1996.

[14] Video Quality Experts Group (VQEG), "Final report from the Video Quality Experts Group on the validation of objective models of video quality assessment, phase II," 2003 VQEG. Available at: www.vqeg.org

[15] ITS Video quality research. Available at: www.its.bldrdoc.gov/n3/video/VQM_software.php

[16] Joint Video Team of ISO/IEC MPEG and ITU-T VCEG, H.264/AVC, Reference Software JM10.1 (online), http://iphome.hhi.de/suehring/tml/download/old_jm/jm10.1.zip

[17] H. J. LichtFuss. Evolution eines Rohrkrümmers. Diplomarbeit, Technische Universität Berlin, 1965

[18] H.-P Schwefel. Kybernetische Evolution aks Strategie der experimentellen Forschung in der Strömungstechnik. Diplomarbeit Technische Universität Berlin, 1965

[19] Charles Darwin, The Origin of Species: By Means ofNatural Selection or the Preservation of Favoured Races in theStruggle for Life (Bantam Classic), Bantam Classics,Reprint. 1999.

[20] Fitzpatrick, J.M., Grefenstette, J.J. and Van-Gucht, D."Image registration by genetic search," Proceedings of Southeastcon 84, Louisville, KY, 460-464, Apr 1984.

[21] Man F. So \& Angus Wu, "Hardware Implementation of Four-Step Genetic Search Algorithm", IEEE Signal Processing Society 1999 Workshop on Multimedia Signal Processing, Copenhagen, Denmark, September 1999.

[22] Xu Yuelei, Bi Duyan and Mao Baixin, “ A Genetic Search Algorithm For Motion Estimation", Proceedings of 5th International Conference on Signal Processing Proceedings, Beijing, China, 2000.

[23] Ingo Rechenberg, Evolutionsstrategie '94. Stuttgart: FrommannHolzboog 1994.

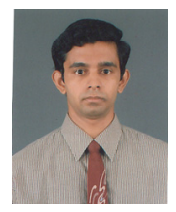

S. L. P. Yasakethu received the BSc Engineering degree (First Class Hons.) in Electrical and Electronic Engineering from the University of Peradeniya Sri Lanka in 2007. He has been awarded a prize for best performance in Electronic Communication Engineering, by the University of Peradeniya. Currently he is a research student completing his $\mathrm{PhD}$ in the Centre for Communication Systems Research (CCSR) in the University of Surrey, UK. His research interests are in Distributed Video Coding and Quality of Experience in Multimedia Communications.

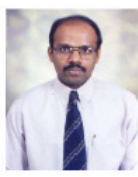

W. A. C. Fernando received the B.Sc. Engineering degree (First class) in Electronic and Telecommunications Engineering from the University of Moratuwa, Sri Lanka in 1995 and the MEng degree (Distinction) in Telecommunications from Asian Institute of Technology (AIT), Bangkok, Thailand in 1997. He completed his $\mathrm{PhD}$ at the Department of Electrical and Electronic Engineering, University of Bristol, UK in February 2001. Currently, he is a senior lecture in signal processing at the University of Surrey, UK. Prior to that, he was a senior lecturer in Brunel University, UK and an assistant professor in AIT. His current research interests include Distribute Video Coding (DVC), 3D video coding, intelligent video encoding for wireless communications, OFDM and CDMA for wireless channels, channel coding and modulation schemes for wireless channels. He has published more than 160 international papers on these areas. He is a senior member of IEEE and a fellow of the HEA, UK. He is also a member of the EPSRC College.

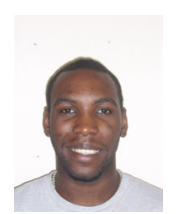

Steven Adedoyin holds a BEng (Hons) in Microelectronics Engineering that was obtained from Brunel University. Currently he is a research student completing his $\mathrm{PhD}$ in the Centre for Communication Systems Research (CCSR) in the University of Surrey, UK. His current research interest lies in 3D integral imaging, 3D video coding, H.264 and Evolutionary Computing.

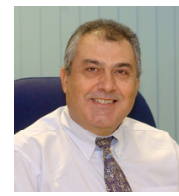

Ahmet M. Kondoz received the B.Sc. (Hons.) degree in engineering, the M.Sc. degree in telematics, and the Ph.D. in communication in 1983, 1984, and 1986, respectively. He became a Lecturer in 1988, a Reader in 1995, and then in 1996, a Professor in Multimedia Communication Systems and deputy director of Centre for Communication Systems Research (CCSR), University of Surrey, Guildford, U.K. He has over 250 publications, including a book on low bit-rate speech coding and several book chapters. He has graduated more than $40 \mathrm{Ph}$.D. students in the areas of speech/image and signal processing and wireless multimedia communications, and has been a consultant for major wireless media terminal developers and manufacturers. Prof Kondoz has been awarded several prizes, the most significant of which are The Royal Television Societies' Communications Innovation Award and The IEE Benefactors Premium Award. He has been on the Refereeing College for EPSRC and on the Canadian Research Councils. He is a member of the IEEE and the IET. 